\title{
Can Moderate Patient Prosthesis Mismatch Be Tolerated in a Selected Group of Patients with Aortic Valve Replacement?
}

\author{
İbrahim Cagri Kaya, MD, ${ }^{1}$ Mustafa Mert Ozgur, $M D,{ }^{2}$ Hakan Hancer, $M D,{ }^{2}$ Tanil Ozer, MD, ${ }^{2}$ \\ Ozge Altas Yerlikhan, $\mathrm{MD},{ }^{2}$ Hasan Sunar, $\mathrm{MD}^{2}$ \\ ${ }^{1}$ Department of Cardiovascular Surgery, Eskisehir City Hospital, Eskisehir, ${ }^{2}$ Department of Cardiovascular Surgery, Kartal Kosuyolu \\ Research and Training Hospital, Istanbul, Turkey
}

\section{ABSTRACT}

Introduction: In this study, we aimed to determine the incidence of patient prosthesis mismatch (PPM) and its effects on ejection fraction (EF), gradients, and late survival.

Methods: 200 patients who underwent isolated mechanical AVR between March 2013 and May 2016 were retrospectively evaluated based on patient records.

Results: 200 patients were included in the study. No PPM was detected in $42(21 \%)$ patients, moderate PPM in 122 $(61 \%)$, and severe PPM in $36(18 \%)$ patients. A significant decrease was found in all groups in terms of mean valve gradients and LVMI (preoperative LVMI compared with postoperative LVMI at the 12 th month) $(P<.001)$. A $30 \%$ decrease in mean LVMI in the no PPM and moderate PPM groups and a $20 \%$ decrease in the severe PPM group were detected at the 6 th month.

Conclusion: In our postoperative data, we found that EF was preserved, the transvalvular gradient reduced, and LVMI decreased. There was no difference in mortality rates between the control (no PPM) group and the moderate PPM group. Taking into account our patient groups, we can say that no-to-moderate PPM has no major effect on left ventricular remodeling in patients with preserved left ventricular functions.

\section{INTRODUCTION}

The term valve prosthesis-patient mismatch (PPM) is a condition of having a small prosthetic heart valve according to the patient's body surface area (BSA). In other words, PPM that develops after aortic valve replacement (AVR) means that the efficient orifice area (EOA) of the inserted valve is inadequate for cardiac output because of residual high transvalvular gradient.

Many studies have been conducted to observe the relationship of PPM with decreased exercise capacity and survival,

Received October 4, 2020; received in revised form December 6, 2020; accepted December 7, 2020

Correspondence: İbrabim Cagri Kaya, MD, Department of Cardiovascular Surgery, Eskisehir City Hospital, Eskisehir, 26080, Turkey; 0000-0002-29094034 (e-mail: icagrikaya@gmail.com). especially in the last decade, but there is still no consensus on this issue [Blackstone 2003]. With the development of third-generation prosthetic valves, positive results have been shown in patients with PPM. On the other hand, some studies advocate that mismatch is a strong and independent marker of short- and long-term mortality after AVR [Blais 2003]. Actually the effect of PPM on mortality is associated with left ventricular function. The degree of preoperative left ventricular hypertrophy also plays an important role in mortality. Many studies have reported the early and late prognostic importance of preoperative left ventricular mass index (LVMI) increase [Zhang 2019; Garcia Fuster 2003].

Studies questioning the effect of PPM on left ventricular functions and mortality have led surgeons to decide whether to take the risks associated with aortic root enlargement techniques and to what extent to tolerate this mismatch. On the basis of these questions the aim of our study was to determine the incidence of PPM and its effects on EF, gradients, and late survival.

\section{METHODS}

\section{Patient Selection}

The data of 1184 patients who underwent aortic interventions between March 2013 and May 2016 were retrospectively evaluated based on patient records. Patients who underwent concomitant cardiac interventions, patients with low ejection fraction $(\mathrm{EF}<50 \%)$, with chronic obstructive pulmonary disease, under 18 years, and those who underwent surgery in emergency conditions were excluded from the study. Reoperations with thrombus on the prosthetic valve, and with paravalvular leakage and prosthetic valve dehiscence in the postoperative period were excluded from the study. Additonally patients who underwent ascending aortic interventions, aortic root enlargement procedures were excluded from the study. In order to evaluate left ventricular reverse remodeling after surgery, we included patients who lived and had preoperative echocardiography (Echo) and postoperative Echos at the 1st, 6th, and 12 th months. Eventually 200 patients who met the inclusion criteria were included in the study. The preoperative demographic data, surgical, and postoperative data of the patients, and the brand and size of the prosthesis inserted 
Table 1. Normal Reference Values of Effective Orifice Areas for the Prosthetic Valves

\begin{tabular}{lcccccc}
\hline & \multicolumn{5}{c}{ No. of patients, $\mathrm{n}(\%)$} \\
\hline & 19 & 21 & 23 & 25 & 27 & 2.98 \\
Carbomedics standard & 134 & 1.00 & 1.54 & 1.63 & 2.10 & 2.50 \\
ATS standard & 22 & 1.20 & 1.50 & 1.70 & $2.10(22)$ & $2.40(24)$ \\
ATS AP & 6 & $1.50(18)$ & $1.70(20)$ & 1.70 & 2.00 & 2.30 \\
Sorin & 30 & 1.40 & 2.00 & 2.40 & 2.50 \\
St. Jude Regent & 8 & 1.50 & & & 3.60 \\
\hline
\end{tabular}

Effective orifice areas are presented as $\mathrm{cm}^{2}$.

into each patient were evaluated.

\section{Echocardiographic Evaluation}

Doppler and 2D echocardiographic data in our hospital database were evaluated for each patient. Ejection fraction (EF), mean valve gradient, left ventricular end-diastolic diameter (LVEDD), interventricular septum diameter (IVS), posterior wall thickness (PWD) values from preoperative echocardiography, and postoperative echocardiography at the 1st, 6 th, and 12 th months were used.

LVMI for each patient was calculated using the formula of Devereux and Reishek:

LVMI $\left(\mathrm{g} / \mathrm{m}^{2}\right):(1.04 \mathrm{X}[(\mathrm{LVEDD}+\mathrm{IVS}+\mathrm{PWD}) 3-$ LVEDD3] - 13.6) / BSA

Values over $134 \mathrm{~g} / \mathrm{m}^{2}$ for men and over $110 \mathrm{~g} / \mathrm{m}^{2}$ for women were considered as left ventricular hypertrophy (LVH) after this calculation.

Left ventricular ejection fraction was determined using a volumetric method and visual estimation. Mean aortic valve gradient was calculated using Doppler echocardiography and aortic valve area continuity equation.

\section{Operative Data}

All surgical records were reviewed to enroll the type, size, and brand of the inserted aortic valve. Cross clamp and cardiopulmonary bypass $(\mathrm{CPB})$ times were also analyzed.

When the cap sizes and brands were taken into consideration, valves sized $19 \mathrm{~mm}$ were inserted into 32 patients, 21 $\mathrm{mm}$ into 80 patients, $23 \mathrm{~mm}$ into 48 patients, $25 \mathrm{~mm}$ into 34 patients, and $27 \mathrm{~mm}$ into 6 patients. The in-vitro reference active orifice areas of the valves obtained from each manufacturer are shown in Table 1.

\section{Surgical Technique}

Only isolated AVR cases were included in the study. Under general anesthesia, operations were performed with standard median sternotomy. Standard aortic arterial cannulation and unicaval venous cannulation was performed to institute cardiopulmonary bypass. Intermittent antegrade cold blood cardioplegia was used for myocardial protection. Annular decalcification was performed for every patient with annular calcifications. Annular sizing was performed via manufacturer's sizers. We used some high performance (larger EOA) valves of particular brands and implanted them supra-annularly (ATS Ap in 6 patients, ST. JUDE REGENT in 8 patients). Other valves were implanted intra-annularly.

\section{Definition and Evaluation of PPM}

Index efficient orifice areas (iEOAs) were obtained by dividing the in vitro reference EOAs determined for each patient into the BSAs of the patients. For patients with iEOA above $0.85 \mathrm{~cm}^{2} / \mathrm{m}^{2}$, PPM was considered as clinically meaningless (no PPM). Patients with iEOA between $0.65 \mathrm{~cm}^{2} / \mathrm{m}^{2}$ to $0.85 \mathrm{~cm}^{2} / \mathrm{m}^{2}$ were considered as moderate, and patients with iEOA $\leq 0.65 \mathrm{~cm}^{2} / \mathrm{m}^{2}$ were considered as severe PPM. In this study when we compare PPM groups, the clinically meaningless (no PPM) group was accepted as the control group.

\section{Statistical Analysis}

All data are expressed as mean \pm standard deviation (SD) for continuous variables and percentage and numbers for categorical variables. In addition to descriptive statistical methods (mean, standard deviation, max, min), one-way variance analysis (ANOVA), the Mann-Whitney $U$ test, and the Kruskal Wallis test were used to evaluate the data. The Tukey multiple comparison test was used for subgroup comparisons. Repeated measures ANOVA was used to compare the variation in repetitive echocardiography follow-up in the same group.

\section{RESULTS}

The mean age of the 126 male and 74 female (total of 200) patients was $59 \pm 16.4$ years. The mean follow-up period of the patients was $25 \pm 10$ months, and the average valve size was $21 \pm 2 \mathrm{~mm} .148$ patients had aortic stenosis, 24 patients had aortic insufficiency, and 18 patients had aortic stenosis + aortic insufficiency.

According to their calculated iEOAs, 42 (21\%) patients had no PPM, $122(61 \%)$ had moderate PPM, and $36(18 \%)$ had severe PPM. The comparison of preoperative data and surgical data of the patients according to PPM is shown in Table 2. We observed that the patients with severe PPM were 
Table 2. Preoperative and Surgical Variables of Patients with Patient-Prosthesis Mismatch (PPM)

\begin{tabular}{|c|c|c|c|}
\hline Mean* & $32-81$ & $25-87$ & $22-73$ \\
\hline Range & $38-4$ & $78-44$ & $10-26$ \\
\hline \multicolumn{4}{|l|}{ BSA (M2) } \\
\hline Mean* & $2 \pm 0.18$ & $1.87 \pm 0.15$ & $1.90 \pm 0.33$ \\
\hline Range & $1.6-2.2$ & $1.5-2.2$ & $1.3-2.3$ \\
\hline $\mathrm{DM}(\mathrm{N})$ & $12(28 \%)$ & $31(25)$ & $14(38)$ \\
\hline STS-PROM & $2.6 \pm 1$ & $3 \pm 1.8$ & $3.2 \pm 2$ \\
\hline CPB time, $\min$ & $51-169$ & $50-304$ & $55-245$ \\
\hline \multirow[t]{2}{*}{ Cross-clamp time, $\min$} & $82.76 \pm 24$ & $76.30 \pm 27$ & $107.33 \pm 47$ \\
\hline & $33-120$ & $28-150$ & $34-208$ \\
\hline Prosthesis size, mm & $24.72 \pm 1.8$ & $21.46 \pm 1.4$ & $20.55 \pm 1.6$ \\
\hline
\end{tabular}

*Values are mean \pm SD; BSA indicates body surface area; CPB, cardiopulmonary bypass time; DM, diabetes mellitus; EU SC II, Euro score II; STS PROM, Society of Thoracic Surgeons-Predicted Risk of Mortality; PPM, prosthesis patient mismatch.

Table 3. Preoperative and Follow-Up Echocardiographic Data

\begin{tabular}{|c|c|c|c|c|c|c|c|c|c|c|c|c|}
\hline $\begin{array}{l}\text { Variable } \\
(\text { mean } \pm s t d)\end{array}$ & No & Mod & Sev & No & Mod & Sev & No & Mod & Sev & No & Mod & Sev \\
\hline LVEF (\%) & $60 \pm 6$ & $61 \pm 6$ & $63 \pm 4$ & $59 \pm 6$ & $57 \pm 7$ & $60 \pm 5$ & $60 \pm 4$ & $60 \pm 5$ & $61 \pm 3$ & $61 \pm 4$ & $62 \pm 4$ & $62 \pm 3$ \\
\hline $\begin{array}{l}\text { Mean gradient } \\
(\mathrm{mm} \mathrm{Hg})\end{array}$ & $45 \pm 11$ & $49 \pm 13.5$ & $54 \pm 16.5$ & $17 \pm 6.5$ & $19 \pm 8.5$ & $25 \pm 10$ & $16 \pm 5.5$ & $18 \pm 7.5$ & $22 \pm 8$ & $14 \pm 5$ & $18 \pm 7$ & $22 \pm 10.5$ \\
\hline
\end{tabular}

LVEF indicates left ventricular ejection fraction; LVMI, left ventricular mass index; Mod, moderate; Sev, severe.

older and their BSA was higher than the patients with moderate PPM. Although the number of female patients was less than male patients, clinically significant PPM (moderate and severe) was detected in $95 \%$ of female patients and $70 \%$ of male patients. The mean aortic cross-clamp times and cardiopulmonary bypass times of the severe PPM group were longer than the moderate PPM group.

Table 3 shows echocardiographic follow-up data of the three patient groups and LVMI values. There was no significant difference between the groups in terms of LVEF $(P>$ $.05)$. The mean valve gradient was significantly higher in the severe PPM group than in the other two groups $(P=.007, P$
$=.026$, and $P=.013$, respectively). There was no difference between the groups in terms of LVEF, LVMI, and mean valve gradient except for LVMI at the postoperative 12th month; it was lower in the control PPM group than in the other groups $(P=.028)$.

When the entire group of patients was evaluated, LVEF was preserved and there was a significant decrease in the mean valve gradient. The decrease in mean valve gradient and LVMI was relatively high in the control PPM group and the decrease in LVMI was relatively low in the severe PPM group (Figures 1 and 2). Despite this difference, significant decrease in the mean valve gradient and in the LVMI at postoperative 


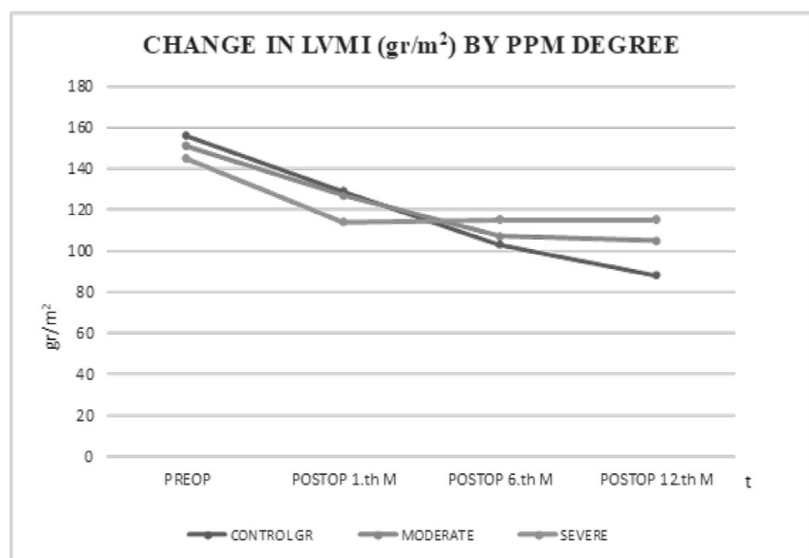

Figure 1. LVMI is significantly decreased in all patient groups in the end of 12 months. The amount of decrease increases when the degree of PPM decreases.

\section{CHANGE IN MEAN GRADIENT ( mmHg ) BY PPM DEGREEE}

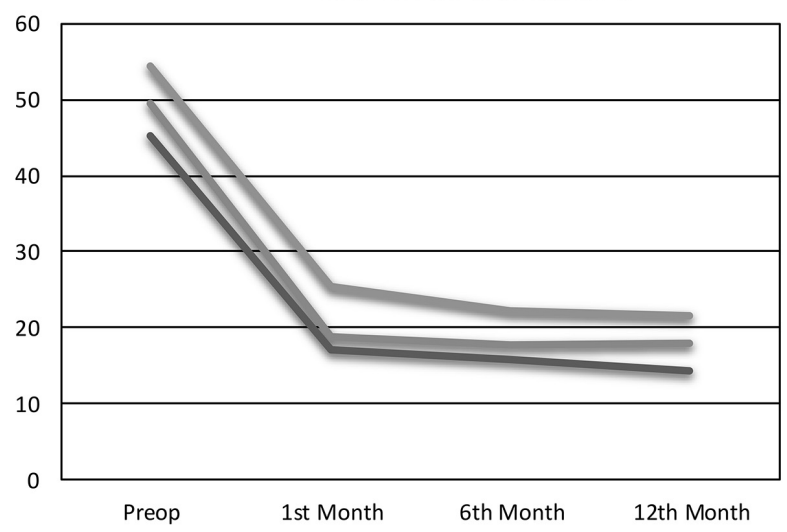

Figure 2. Mean gradient is significantly decreased in all patient groups in the end of 12 months. The amount of decrease increases when the degree of PPM decreases.

12th month in all three groups was noticeable $(P<.001 / P<$ $.001 / P=.03)$.

Total mortality rate was $17 \% .4 \%$ of these patients were in the control PPM group, $4 \%$ in the moderate PPM group, and $9 \%$ in the severe PPM group. In the log rank analysis there was no significant difference between the control group and moderate PPM group $(P=.806)$, but there was a significant difference between the control group and severe PPM group $(P=.036)$ (Figure 3).

\section{jDISCUSSION}

Successful AVR is generally defined as improvement in left ventricular function after surgery (no decrease in LVEF in patients with preserved ventricular functions), decrease in

\section{Kaplan-Meier survival estimates}

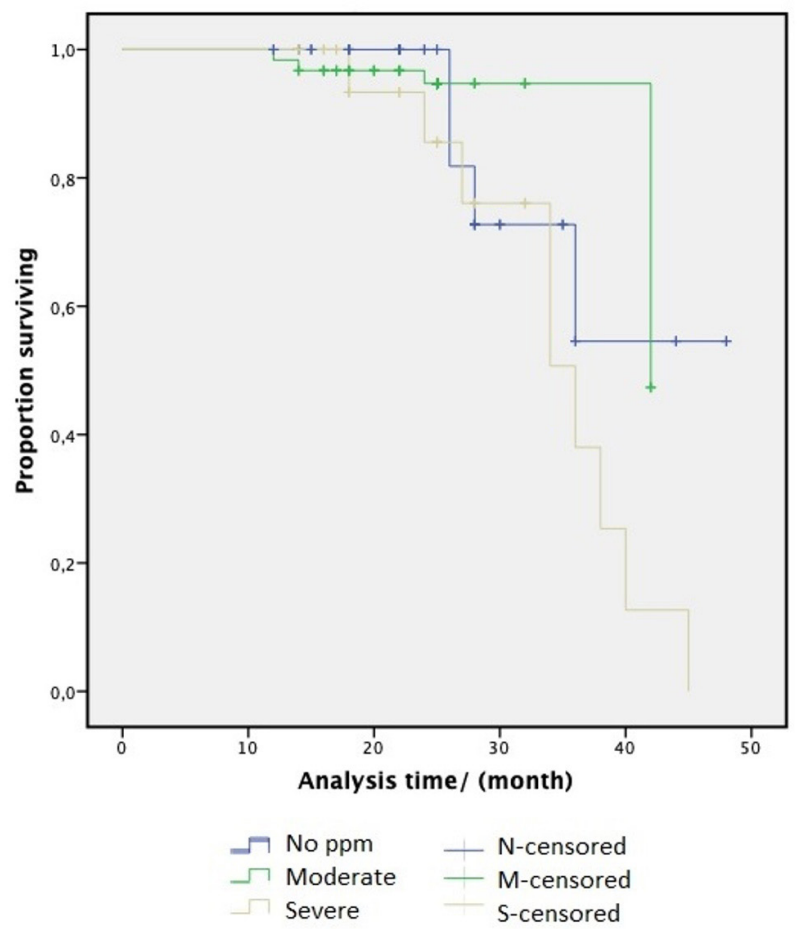

Figure 3. Kalan-Meier survival estimates.

previously increased left ventricular mass, improved exercise capacity, and increased life span after surgery [Vipparth 2020]. When a prosthetic valve with a small EOA compared with the patient's body size is inserted, high pressure differences may occur in the postoperative period, which may reduce the success of the surgery.

PPM was first described by Rahimtoola in 1978 as "smaller EOA of aortic prosthetic valve than one's natural valve" [Maheshwari 2020]. Many studies have been conducted about PPM to the present day. Most experts argue that PPM is manifested by the lack of expected increase in postoperative exercise capacity and high mortality rates [Swinkels 2016]. With the use of new-generation valves with high performance and the increase in the number of studies performed on welldefined patient groups, some studies suggest that despite high PPM rates, postoperative mortality rates are lower and quality of life is higher than expected [Hoffmann 2018].

In the literature, moderate PPM rates are stated as 20-70\% and severe PPM rates as 2-11\% [Bilkhu 2019; Otto 2020]. In our study, $61 \%$ of patients had moderate and $18 \%$ had severe PPM. Severe PPM rates were relatively high but we think that it was related to relatively higher BSA rates in our patients. Guo et al observed that moderate and severe PPM accounted for $20 \%$ of patients in their study. In the same study, the mean BSA of the patients was $1.6 \mathrm{~kg} / \mathrm{m}^{2}$, whereas the mean BSA of our patient population was $1.9 \mathrm{~kg} / \mathrm{m}^{2}$ [Guo 2017].

We used projected IEOA values to define PPM. Projected IEOA is compatible with postoperative rest and exercise 
Table 4. Postoperative 12th month LVMI $\left(\mathrm{g} / \mathrm{m}^{2}\right)$

\begin{tabular}{lcccc}
\hline PPM & Sex & Mean & $\mathrm{n}$ & Std. Dev \\
\hline No & $\mathrm{M}$ & 91.6 & 19 & 12.8 \\
& $\mathrm{~F}$ & 54.7 & 2 & 0.0 \\
Moderate & Total & 88.1 & 21 & 16.4 \\
& $\mathrm{M}$ & 107.6 & 39 & 34.5 \\
Severe & $\mathrm{F}$ & 102.2 & 22 & 25.6 \\
& Total & 105.7 & 61 & 31.4 \\
& $\mathrm{M}$ & 98.0 & 5 & 7.2 \\
& $\mathrm{~F}$ & 121.8 & 13 & 13.6 \\
\hline
\end{tabular}

LVMI indicates left ventricular mass index; PPM, prosthesis patient mismatch; Std Dev, standard deviation.

transprosthetic gradient and it can be used with $73 \%$ sensitivity and $80 \%$ specificity in patients with high postoperative gradient and suspected mismatch [Bakhtiary 2007; Sakamoto 2010]. Rabus et al reported that age over 70 years, female sex, and severe PPM were independent predictors of early mortality in their study with 701 patients. They also found no association between moderate PPM and mortality [Rabus 2009]. Nozohoor et al showed that 54\% of 1568 patients who underwent AVR were in the moderate or severe PPM group, but they were not able to show an association between PPM and early and late term poor results [Nozohoor 2007]. Okamura et al compared the preoperative and postoperative echocardiographic data of the patients who underwent mechanical aortic valve replacement due to aortic stenosis in terms of LVMI regression, transvalvular gradient, and LVEF. They showed that postoperative LVMI regression, LVEF, and transvalvular gradient follow-up data were similar for both groups of patients with and without PPM [Okamura 2020]. In our study, there was no difference between the control and moderate PPM groups in terms of decreased LVMI, mean valve gradient, and LVEF values in the postoperative followup (Table 3, Figures 1 and 2).

The change in LVEF after AVR reflects left ventricular function. Preservation of normal ejection fraction following valve replacement indicates adequate cardiac protection during cardiopulmonary bypass. The regression of left ventricular hypertrophy after surgery indicates a normofunctional valve, which ensures the continuation of normal ventricular function. All these indicate the success of the surgery performed [Monin 2007]. In our study, there was no statistically significant difference between the three groups (control, moderate, and severe PPM) in terms of preoperative LVEF and postoperative LVEF at 1st, 6th and 12th months (the $\mathrm{P}$ values of comparison of preoperative LVEF and postoperative 12 th month LVEF in the three groups were $0.184,0.430$ and 0.454 , respectively). Recovery of functional capacity-ejection fraction and low long-term cardiac mortality after AVR are important for the success of the operation.
Insufficient regression in left ventricular mass is the main cause of cardiac arrhythmia and diastolic dysfunction which are the main causes of mortality [Das De 2019]. LVH was defined as LVMI $>134 \mathrm{~g} / \mathrm{m}^{2}$ in men and $>110 \mathrm{~g} / \mathrm{m}^{2}$ in women [Levy 1987]. In our study, the preoperative mean LVMI of our patients was $151 \mathrm{~g} / \mathrm{m}^{2} ; 300 \mathrm{~g} / \mathrm{m}^{2}$ in men and $148 \mathrm{~g} / \mathrm{m}^{2}$ in women. LVH developed in 152 (76\%) of our patients. LVH was improved in all subgroups (except 26 female patients with severe PPM) (Table 4).

Centers that advocate that moderate and severe PPM has negative effects on early and late mortality, as well as functional capacity, suggest not to avoid aortic root enlargement techniques if there is high probability of moderate or severe PPM in the postoperative period [Sá 2019a]. In contrast, Kitamura et al compared 28 aortic root enlargement techniques (13 nick, 12 manouguian and 3 apicoaortic bypasses) and 17 standard AVRs and showed that patients with aortic root enlargement had higher long-term mortality and morbidity rates. Longer aortic cross-clamp and CPB durations, more perioperative bleeding, and higher rates of bleedingrelated reoperation were shown as the reasons for high mortality and morbidity [Kitamura 2013]. Some recent studies comparing concomitant aortic root enlargement with classical AVR showed no significant difference in mortality and also showed that isolated AVR patients had better results in terms of operation duration, postoperative respiratory complications, etc. [Haunschild 2019; Kim 2020].

Mascherbauer et al failed to show a relationship between PPM and early and late mortality in their study [Mascherbauer 2008]. In the same study, PPM was detected in 54\% of patients and most were women, symptomatic, elderly, had high EuroSCOREs, and had concomitant coronary artery disease. The study specifically underlined that moderate PPM rates were very high, but this had no effect on mortality rates; therefore, it would not be right to recommend complex procedures in patients undergoing valve replacement due to isolated severe aortic stenosis. Patients with PPM are usually older patients with high-risks and high euroSCOREs, which leads surgeons to keep surgery to a short duration. Given that aortic root enlargement techniques add at least 20 minutes to the aortic cross-clamp time, the idea that moderate PPM can be tolerated is strengthened [Mascherbauer 2008].

Vriesendorp et al argue that lack of strict criteria for aortic root dilatation could affect results because subjective observation and evaluation of aortic root anatomy, comorbidities, and the surgeon's experience of complex procedures could potentially affect the decision-making process for root expansion. In the same study, it was emphasized that the smaller mean valve size implanted in the ARE (aortic root enlargement) group was confusing, and no difference was observed in the ARE group in 5-year survival [Vriesendorp 2020]. Hawkins et al showed that there was higher mortality and morbidity rates and longer hospital stay in the concomitant ARE group in their study with 6046 patients. What is more interesting is that there was no significant difference in PPM rates between the two groups [Hawkins 2019].

Sá MPBO et al evaluated 10 studies involving 13,174 patients with and without aortic root enlargement in a 
meta-analysis and found that the mean aorta cross-clamp duration was significantly longer and perioperative mortality was significantly higher in the aortic root enlargement group [Sá 2019b].

In patients undergoing isolated mechanical AVR, transvalvular gradient, regression in LVMI, and LVEF are important in evaluating the success of the operation. It has been shown that protection of EF affects functional capacity and LVH affects mortality. Although we found high rates of moderate and severe PPM in our study, our follow-up results were very good in terms of the criteria of operational success. Although many studies have been conducted about effects of PPM, controversy still remains due to the fact that the concept of PPM is not uniformly defined, the groups of patients are not explicitly stated and are very heterogeneous, and the causes of death are not discussed.

The main limitations of our study were that it was a single-center, retrospective study and that the postoperative functional capacities of our patients were not included in the study. Given the higher number of patients but heterogeneous groups of patients in the literature, we think that our relatively small but selected group of patients was an advantage of our study

\section{Conclusion}

Our moderate PPM rate was high. However, when we examined our postoperative data, we found that EF was preserved, the transvalvular gradient reduced, and LVMI decreased. There was no difference in mortality rates between the control group and the moderate PPM group. When we evaluate our patient groups, we can say that control-tomoderate PPM has no effect on left ventricular remodeling in patients with preserved left ventricular functions $(\mathrm{EF}>50)$. When the risks of aortic root enlargement techniques such as duration of operation, perioperative bleeding, postoperative respiratory complications, and the individual risks of this group of patients (e.g. age, sex, calcific aortic root) are considered, we argue that moderate PPM can be tolerated.

\section{REFERENCES}

Bakhtiary F, Schiemann M, Dzemali O, et al. 2007. Impact of patientprosthesis mismatch and aortic valve design on coronary flow reserve after aortic valve replacement. J Am Coll Cardiol 49:790-6.

Bilkhu R, Jahangiri M, Otto CM. 2019. Patient-prosthesis mismatch following aortic valve replacement. Heart 105(Suppl2):s28-s33.

Blackstone EH, Cosgrove DM, Jamieson WR, et al. 2003. Prosthesis size and long-term survival after aortic valve replacement. J Thorac Cardiovasc Surg 126:783-96.

Blais C, Dumesnil JG, Baillot R, Simard S, Doyle D, Pibarot P. 2003. Impact of valve prosthesis-patient mismatch on short-term mortality after aortic valve replacement. Circulation 108:983-8.

Das De SD, Nanjappa A, Morcos K, et al. 2019. The effect of patientprosthesis mismatch on survival after aortic and mitral valve replacement: a 10 year, single institution experience. J Cardiothorac Surg 14:214.

Garcia Fuster R, Montero Arguto JA, Gil Albarova O, et al. 2003. Left ventricular mass index in aortic valve surgery: a new index for early valve replacement? Eur J Cardiothorac Surg 23:696-702.

Guo L, Zheng J, Chen L, et al. 2017. Impact of prosthesis patient mismatch on short-term outcomes after aortic valve replacement: a retrospective analysis in East China. J Cardiothorac Surg 12:42.

Haunschild J, Scharnowski S, Mende M, et al. 2019. Aortic root enlargement to mitigate patient-prosthesis mismatch: do early adverse events justify reluctance? Eur J Cardiothorac Surg pii:ezz016.

Hawkins RB, Beller JP, Mehaffey JH, et al. 2019. Incremental risk of annular enlargement: a multi-institutional cohort study. Ann Thorac Surg 108:1752-9.

Hoffmann G, Abraham-Westphal S, Attmann T, et al. 2018. Impact of patient-prosthesis mismatch following aortic valve replacement on longterm survival and quality of life. Thorac Cardiovascular Surg.

Kim H, Lee S, Joo H, et al. 2020 Impact of suture techniques for aortic valve replacement on prosthesis-patient mismatch. Ann Thorac Surg 109:661-7.

Kitamura T, Torii S, Hanayama N, et al. 2013. Moderate prosthesispatient mismatch may be negligible in elderly patients undergoing conventional aortic valve replacement for aortic stenosis. Int Heart J 54:11-14.

Levy D, Savage DD, Garrison RJ, Anderson KM, Kannel WB, Castelli WP. 1987. Echocardiographic criteria for left ventricular hypertrophy: the Framingham Study. Am J Cardiol 59:956-60.

Maheshwari A, Gupta R, Saha D, Naqvi SEH, Minhas HS, Geelani MA. 2020. Konno procedure for managing small aortic root during aortic valve replacement surgery: an experience of 12 cases. World Journal Cardiovasc Surg 10:24.

Mascherbauer J, Rosenhek R, Fuchs C, et al. 2008. Moderate patientprosthesis mismatch after valve replacement for severe aortic stenosis has no impact on short- and long term mortality. Heart 94:1639-45.

Monin J-L, Monchi M, Kirsch ME, et al. 2007. Low-gradient aortic stenosis: impact of prosthesis-patient mismatch on survival. Eur Heart J 28:2620-6.

Nozohoor S, Nilsson J, Luhrs C, Roijer A, Sjogren J. 2007. The influence of patientprosthesis mismatch on in-hospital complications and early mortality after aortic valve replacement. J Heart Valve Dis 16:475-82.

Okamura H, Hori D, Kusadokoro S, et al. 2020. Long-term outcomes and echocardiographic data after aortic valve replacement with a $17-\mathrm{mm}$ mechanical valve. Circ J CJ-20.

Otto ME, Atik F, Moreira M, et al. 2020. Determinants of aortic prosthesis mismatch in a Brazilian Public Health System hospital: Big patients or small prosthesis? Arquivos brasileiros de cardiologia 114:12-22.

Rabus MB, Kirali K, Kayalar N, et al. 2009. Effects of patient-prosthesis mismatch on postoperative early mortality in isolated aortic stenosis. J Heart Valve Dis 18:18-27.

Sá MPBDO, de Carvalho MMB, Sobral Filho DC, et al. 2019. Surgical aortic valve replacement and patient-prosthesis mismatch: a meta-analysis of 108,182 patients. Euro J Cardiothorac Surg 56:44-54.

Sá MPBO, Carvalho MMB, Filho DCS, et al. 2019. Impact of surgical aortic root enlargement on the outcomes of aortic valve replacement: a meta-analysis of 13,174 patients. Interactive Cardiovasc Thorac Surg 29:74-82.

Sakamoto Y, Yoshitake M, Naganuma H, Kawada N, Kinouchi K, Hashimoto K. 2010. Reconsideration of patient-prosthesis mismatch definition from the valve indexed effective orifice area. Ann Thorac Surg 89:1951-5. 
Swinkels BM, Bas A, Kelder JC, Vermeulen FE, Jurriën M. 2016. Prosthesis-patient mismatch after aortic valve replacement: effect on longterm survival. Ann Thorac Surg 101:1388-94.

Vipparth SC, Ravi V, Avula S, et al. 2020. Meta-analysis of transcatheter aortic valve implantation versus surgical aortic valve replacement in patients with low surgical risk. Am J Cardiol 125:459-68.
Vriesendorp MD, de Lind van Wijngaarden R, Klautz R. 2020. Concomitant aortic root enlargement is perhaps safe, but is it also effective? Euro J Cardiothorac Surg 57:815.

Zhang HW, Gu J, Xiao ZH, et al. 2019. Global longitudinal strain in prosthesis-patient mismatch: relation to left ventricular mass regression and outcomes. J Cardiovasc Med 20: 434-41. 